\title{
Universiteit
}

Leiden

The Netherlands

\section{Route towards the ideal thresholdless laser}

Dutra, S.M.; Woerdman, J.P.; Visser, J.; Nienhuis, G.

\section{Citation}

Dutra, S. M., Woerdman, J. P., Visser, J., \& Nienhuis, G. (2002). Route towards the ideal thresholdless laser. Physical Review A, 65, 033824. doi:10.1103/PhysRevA.65.033824

Version: $\quad$ Not Applicable (or Unknown)

License: $\quad$ Leiden University Non-exclusive license

Downloaded from: https://hdl.handle.net/1887/61359

Note: To cite this publication please use the final published version (if applicable). 


\title{
Route toward the ideal thresholdless laser
}

\author{
S. M. Dutra, J. P. Woerdman, J. Visser, and G. Nienhuis \\ Huygens Laboratory, Leiden University, P.O. Box 9504, 2300 RA Leiden, The Netherlands
}

(Received 14 September 2001; published 26 February 2002)

\begin{abstract}
We consider what happens to a laser when all incoherent processes are reduced to the minimum needed to keep emission irreversible. Specifically, we investigate the case where the vacuum Rabi frequency is larger than any decay rate in the laser except for the atomic polarization decay rate. Using a fully quantum description, we show that this laser can be made to go continuously from a regime with a well-defined threshold to the ideal thresholdless regime, where the photon statistics is always Poissonian even for arbitrarily small pump powers. We suggest how a proof-of-principles experiment can be realized in the microwave domain.
\end{abstract}

DOI: 10.1103/PhysRevA.65.033824

PACS number(s): 42.50.Lc, 03.65.Yz, 42.55.Ah

\section{INTRODUCTION}

The simplest scenario for the interaction between electromagnetic radiation and matter is a single mode of the radiation field interacting with a two-level system. This is described by the celebrated Jaynes-Cummings model [1] whose list of amazing predictions includes the transformation of spontaneous emission into a reversible process: a quantum Rabi oscillation. This simple scenario has been realized in micromaser experiments [2], where a well-known consequence of having coherent Rabi oscillations is the creation of trapping states [3] in which the field is highly nonclassical. A laser, on the other hand, is a much more complicated system. The fundamental interaction between atom and radiation field can still be described by the Jaynes-Cummings model, but in addition there are incoherent dissipative processes responsible for the decay of the cavity field, of the atomic polarization, and of the atomic populations. These incoherent processes destroy most of the interesting quantum effects produced by the coherent Jaynes-Cummings interaction. The resulting dynamics depends on how these decay rates compare with each other and, in general, can be rather complicated.

Here we discuss what happens when all these additional complications are removed leaving only the essential feature that distinguishes a laser from a micromaser: the irreversibility of emission. We show that this simple laser can operate in the so-called ideal thresholdless regime [4], where the photon statistics is Poissonian for all pump powers. The ideal thresholdless regime has never been reached in the laboratory. We indicate how a proof-of-principles realization of this regime can be achieved in the microwave domain. Our discussion also gives rise to a simple theoretical model, where the transition between ordinary threshold laser operation and the ideal thresholdless regime can be studied analytically, without any adiabatic elimination, and within a full quantum theory of matter and field.

In order to put this into perspective, we note that a key parameter in the threshold behavior of a laser is $\beta$, the fraction of spontaneous emission that goes into the lasing mode [5]. When $\beta$ approaches 1, the kink in the input-output curve that signals the laser threshold disappears [6]. For this reason, lasers with $\beta \rightarrow 1$ have been called thresholdless. The very notion of threshold, however, becomes rather controver- sial when $\beta$ approaches $1[4,7]$. An important aspect behind this controversy is the photon statistics. As above the threshold region the photon statistics of an ordinary laser is Poissonian, one expects the thresholdless regime to be characterized by Poissonian photon statistics for all pump powers. However, contrary to expectations, having $\beta \rightarrow 1$ does not guarantee such ideal thresholdless behavior. In fact, the conditions for achieving the ideal thresholdless regime of Poissonian photon statistics at any pump power have been determined by Rice and Carmichael [4]. Assuming the adiabatic limit, where the decay of the atomic polarization $\gamma_{\perp}$ is much larger than all the other rates in the laser, they derived as conditions, not only that $\beta \rightarrow 1$, but also that the cavity decay rate $\kappa$ be much smaller than the spontaneous emission rate in the mode. This implies that the vacuum Rabi frequency $g$ must satisfy $g \gg \kappa, \gamma_{\|}$, where $\gamma_{\|}$is the atomic (population) decay rate. Unfortunately, at present, for optical transitions $g$ can be made only slightly larger than $\kappa$ and $\gamma_{\|}$[8]. In the microwave regime, on the other hand, this condition is readily fulfilled, making a proof-of-principles experiment possible.

In the next section we introduce our simple laser model. Then in Sec. III, we discuss the transition between ordinary threshold laser operation and the ideal thresholdless regime. In Sec. IV, we show that this thresholdless regime can also be reached in an ordinary micromaser without any atomic beam velocity selection. Finally, we summarize our conclusions in Sec. V.

\section{IRREVERSIBLE EMISSION: THE ESSENCE OF A LASER}

In a laser, coherent Jaynes-Cummings Rabi oscillations become irreversible emission events because of the stochasticity derived from all those incoherent processes that lead to the decay of the atomic polarization, population, and cavity field. Of these, the largest is usually the atomic polarization decay. As a sufficient amount of stochasticity is all that is required to make emission irreversible, we consider here a very simple laser, where only the atomic polarization is subjected to an incoherent process (a dephasing of the atomic wave functions caused, for example, by collisions). To realize such a laser, the field and the atomic population decay rates must be made much smaller than the vacuum Rabi 
frequency. As these low decay rates are not achievable in the optical regime at the moment, we consider a proof-ofprinciples microwave implementation for concreteness. The suggested implementation involves only a slight modification to the usual micromaser setup [2]. In a micromaser, Rydberg atoms in a beam with negligible velocity spread go, one at a time, through a high- $Q$ superconducting cavity. A micromaser operates in the strong coupling regime where $g$ $\gg \gamma_{\|}, \gamma_{\perp}, \kappa$. To make $\gamma_{\perp}$ non-negligible in comparison with $g$, we introduce dephasing that can be produced in the following way. Many microwave cavity QED experiments have a setup similar to the micromaser but use circular Rydberg atoms and an open (Fabry-Pérot) cavity, where a static electric field is applied across the cavity's superconducting mirrors [9]. Manipulating this electric field, one can produce a Stark broadening of the atomic transition (see, for example, pp. 108-111 of Ref. [10]). This will generate the atomic polarization decay required.

The general theory incorporating incoherent atomic and cavity decay into the Jaynes-Cummings dynamics was developed by Briegel and Englert [11] using damping bases. However, the case considered here, where there is only polarization decay, allows a much simpler treatment using JaynesCummings energy eigenstates as a basis instead.

The master equation for a single atom crossing the cavity, and undergoing atomic polarization damping at the same time, is given by

$$
\frac{\partial}{\partial t} \hat{\rho}=-\frac{i}{\hbar}[\hat{H}, \hat{\rho}]+\mathcal{L} \hat{\rho},
$$

where $\hat{H}$ is the Jaynes-Cummings Hamiltonian

$$
\hat{H}=\hbar \omega \hat{a}^{\dagger} \hat{a}+\hbar \frac{\omega}{2} \hat{\sigma}_{z}+\hbar g\left(\hat{a}^{\dagger} \hat{\sigma}+\hat{\sigma}^{\dagger} \hat{a}\right),
$$

and

$$
\mathcal{L} \hat{\rho}=\frac{\gamma_{\perp}}{2}\left(\hat{\sigma}_{z} \hat{\rho} \hat{\sigma}_{z}-\hat{\rho}\right)
$$

is the Lindblad term describing the phase-damping processes responsible for the decay of the atomic polarization, with $\hat{\sigma}_{z}, \hat{\sigma}$, and $\hat{\sigma}^{\dagger}$ being the usual Pauli matrices.

The eigenstates of the Jaynes-Cummings Hamiltonian are given by

$$
|n \pm\rangle=\frac{1}{\sqrt{2}}(|\uparrow\rangle|n\rangle \pm|\downarrow\rangle|n+1\rangle),
$$

where $|n\rangle$ are photon number states and $|\uparrow\rangle,|\downarrow\rangle$ represent the upper and lower atomic energy eigenstates, respectively. Using $|n \pm\rangle$ as a basis, we find from Eq. (1) the following equation of motion for the matrix elements of $\hat{\rho}$ :

$$
\begin{aligned}
\dot{\rho}_{n_{-s}, m_{-u}}= & -\left\{\frac{\gamma_{\perp}}{2}+i[(n-m) \omega-(s \sqrt{n+1}\right. \\
& -u \sqrt{m+1}) g]\} \rho_{n_{-s}, m_{-u}}+\frac{\gamma_{\perp}}{2} \rho_{n_{s}, m_{u}},
\end{aligned}
$$

where $s$ and $u$ stand for \pm , with $-s$ and $-u$ being the corresponding opposite signs. Equation (5) actually describes a system of coupled differential equations, from which, after some lengthy but straightforward calculations, we can derive the following solution:

$$
\begin{aligned}
\rho_{n_{-s}, m_{-u}}\left(t_{0}+\tau\right)= & \frac{e^{-\left[\gamma_{\perp} / 2+i(n-m) \omega\right] t}}{2}\left\{\cosh \left(\Omega_{n, m, s u} \tau\right)\right. \\
& +\frac{\gamma_{\perp} / 2-i(s \sqrt{n+1}-u \sqrt{m+1}) g}{\Omega_{n, m, s u}} \\
& \left.\times \sinh \left(\Omega_{n, m, s u} \tau\right)\right\} \rho_{n, m}\left(t_{0}\right),
\end{aligned}
$$

where $t_{0}$ is the time the atom enters the cavity, $\tau$ is the interaction time, and

$$
\Omega_{n, m, s u}=\sqrt{\left(\frac{\gamma_{\perp}}{2}\right)^{2}-(\sqrt{n+1}-s u \sqrt{m+1})^{2} g^{2}},
$$

with $s u$ being the product of the two signs.

At this point, it is interesting to use Eq. (6) to calculate the expectation value of the atomic inversion for an excited atom interacting with the cavity vacuum field, so that we can see the effect of the atomic polarization decay on spontaneous emission. With the ordinary Jaynes-Cumming interaction, without any atomic polarization decay, it is well known that the expectation value of the inversion is given by $\left\langle\hat{\sigma}_{z}(t)\right\rangle$ $=\cos (2 g t)$. For the present case, a straightforward calculation reveals that

$$
\left\langle\hat{\sigma}_{z}(t)\right\rangle=\frac{\left(\mathrm{Y}_{-}+\gamma_{\perp}\right) e^{\mathrm{Y}_{-} t}-\left(\mathrm{Y}_{+}+\gamma_{\perp}\right) e^{\mathrm{Y}_{+} t}}{\mathrm{Y}_{-}-\mathrm{Y}_{+}},
$$

where

$$
\mathrm{Y}_{ \pm}=-\frac{\gamma_{\perp}}{2} \pm \Omega_{0,0, \pm}
$$

An interesting limiting case is when the polarization decay rate $\gamma_{\perp}$ is much larger than the vacuum Rabi frequency $g$. Then spontaneous emission becomes completely irreversible, with Eq. (8) reducing to

$$
\left\langle\hat{\sigma}_{z}(t)\right\rangle=\exp \left(-4 \frac{g^{2}}{\gamma_{\perp}} t\right) .
$$

There are two peculiar things about Eq. (10) that we would like to point out. First, instead of approaching -1 for $t$ $\rightarrow \infty$, as does ordinary spontaneous emission in free space where the atom decays completely, Eq. (10) approaches 0 . 
Second, the time scale of the exponential decay in Eq. (10), $\gamma_{\perp} /\left(4 g^{2}\right)$, is much longer than the time scale of reversible emission, $1 / g$. The physical reason for this is that a shift of $\pi$ of the relative phase between the atomic dipole and the field reverses the flow of energy between dipole and field, a feature that is even present in the semiclassical Bloch equations. Without dephasing there is no polarization decay and the Bloch vector describes a great circle on the Bloch sphere passing through the north pole (Rabi oscillation). With dephasing, the Bloch vector also diffuses in the azimuthal direction as it tries to perform a Rabi oscillation. Now every time it diffuses through an angle of $\pi$, the motion on the great circle is reversed (i.e., it starts going back to the excited state, if it was originally going toward the ground state). This constant reversal of the energy flow before the atom can perform a complete Rabi cycle explains the slow rate of energy decay as well as why the steady state occurs with the atom half excited, rather than in the ground state.

We can now convert from the density matrix in the Jaynes-Cummings model dressed basis given by Eq. (6) to the density matrix in the bare basis and then trace over the atomic states to obtain the reduced density matrix for the field in the Fock state basis $\rho_{n, m}$,

$$
\begin{aligned}
\rho_{n, m}\left(t_{0}+\tau\right)= & \frac{e^{-\left[\gamma_{\perp} / 2+i(n-m) \omega\right] \tau}}{2}\left\{\left[\phi_{n, m,+}(\tau)\right.\right. \\
& \left.+\phi_{n, m,-}(\tau)\right] \rho_{n, m}\left(t_{0}\right)+\left[\phi_{n-1, m-1,+}(\tau)\right. \\
& \left.\left.-\phi_{n-1, m-1,-}(\tau)\right] \rho_{n-1, m-1}\left(t_{0}\right)\right\},
\end{aligned}
$$

where

$$
\phi_{n, m, s}(\tau)=\cosh \left(\Omega_{n, m, s} \tau\right)+\frac{\gamma_{\perp} / 2}{\Omega_{n, m, s}} \sinh \left(\Omega_{n, m, s} \tau\right)
$$

According to ordinary micromaser theory, the reduced density matrix $\hat{\rho}\left(t_{i+1}\right)$ for the cavity field in a micromaser (at zero temperature and with Poissonian pumping) after the passage of the $(i+1)$ th atom is related to the the reduced density matrix $\hat{\rho}\left(t_{i}\right)$ after the passage of the $i$ th atom by the mapping [10]

$$
\hat{\rho}\left(t_{i+1}\right)=\left(1-\frac{1}{R} \mathcal{L}_{\text {cav }}\right)^{-1} \mathcal{F}(\tau) \hat{\rho}\left(t_{i}\right)
$$

where $R$ is the injection rate,

$$
\mathcal{L}_{\text {cav }} \hat{\rho}=-\frac{\kappa}{2}\left\{\hat{a}^{\dagger} \hat{a} \hat{\rho}+\hat{\rho} \hat{a}^{\dagger} \hat{a}-2 \hat{a} \hat{\rho} \hat{a}^{\dagger}\right\}
$$

is the Lindblad term that describes cavity losses at zero temperature, and $\mathcal{F}$ is the superoperator that changes $\hat{\rho}$ to its new value after the passage of a single atom. In the ordinary micromaser, $\mathcal{F}$ is obtained from the reduced density matrix of the field undergoing a simple Jaynes-Cummings time evolution. Here $\mathcal{F}$ is given by the combined Jaynes-Cummings time evolution and simultaneous atomic dephasing. Equation (11) gives $\langle n|\mathcal{F}(\tau) \hat{\rho}| m\rangle$ for this case, and using it in Eq. (13), we obtain the following equation for the "steady state" of our laser in the interaction picture:

$$
\begin{gathered}
\frac{R}{2} e^{-\left(\gamma_{\perp} / 2\right) \tau}\left\{\left[\phi_{n, m,+}(\tau)+\phi_{n, m,-}(\tau)-2 e^{\left(\gamma_{\perp} / 2\right) \tau}\right] \rho_{n, m}\right. \\
\left.+\left[\phi_{n-1, m-1,+}(\tau)-\phi_{n-1, m-1,-}(\tau)\right] \rho_{n-1, m-1}\right\} \\
=\frac{\kappa}{2}\left\{(n+m) \rho_{n, m}-2 \sqrt{(n+1)(m+1)} \rho_{n+1, m+1}\right\} .
\end{gathered}
$$

\section{FROM ORDINARY THRESHOLD OPERATION TO THE IDEAL THRESHOLDLESS REGIME}

Applying the standard procedures used in micromaser theory [10] to Eq. (15), we find that the probability of having $n$ photons in the cavity mode in the "steady state" is given by

$$
p_{n}=p_{0} \prod_{k=1}^{n} \frac{N_{\mathrm{ex}}}{2 k}\left(1-G_{k}\right),
$$

where $N_{\mathrm{ex}} \equiv R / \kappa$ is the average number of atoms that cross the cavity within a photon lifetime, $p_{0}$ is the probability of finding no photons in the cavity mode (obtained as a normalization constant from the requirement $\Sigma p_{n}=1$ ), and $G_{k}$, which describes the combined effects of the JaynesCummings evolution and dephasing, is given by

$$
G_{k}=e^{-\Gamma W}\left\{\frac{\Gamma}{\sqrt{\Gamma^{2}-k}} \sinh \left(W \sqrt{\Gamma^{2}-k}\right)+\cosh \left(W \sqrt{\Gamma^{2}-k}\right)\right\},
$$

with $\Gamma \equiv \gamma_{\perp} /(4 g)$ and $W \equiv g \tau$. Now assuming that $\Gamma^{2}$ is sufficiently large so that the pumping can never be intense enough to achieve average photon numbers of the order of $\Gamma^{2}$, i.e., $\Gamma^{2} \gg\langle n\rangle$ where $\langle n\rangle$ is the average photon number, we can safely expand the square root appearing in Eq. (17) in powers of $k / \Gamma^{2}$. For the fraction we keep only the zeroth order term in this expansion, but for the exponentials we keep both the zeroth and first order terms. Then

$$
G_{k}=\exp \left(-k \frac{W}{\Gamma}\right)
$$

From Eqs. (16) and (18), we see that for weak pumping, where $N_{\mathrm{ex}} \ll \Gamma / W$, we can expand Eq. (18) in a power series of $n W / \Gamma$ keeping only the zeroth and the first order terms. Then $p_{n} \approx\left[N_{\mathrm{ex}} W /(4 \Gamma)\right]^{n} p_{0}$ so that the photon statistics is thermal. For strong pumping, where $N_{\mathrm{ex}} \gg \Gamma / W$, Eq. (18) will be vanishingly small making the photon statistics given by Eq. (16) become Poissonian. This is just like an ordinary laser, with the laser threshold given by $N_{\mathrm{ex}}=4 \Gamma / W$. Now, if $W / \Gamma \gg 1, G_{k}$ in Eq. (16) will be vanishingly small for all 


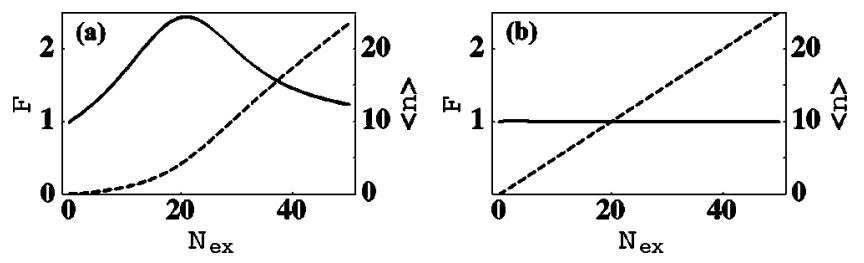

FIG. 1. The Fano parameter (full line) and the average photon number (dotted line) as functions of $N_{\mathrm{ex}}$ for (a) $\Gamma=10, W=2.36$ and (b) $\Gamma=1.8, \quad W=11$. The Fano parameter $F=\left(\left\langle n^{2}\right\rangle\right.$ $\left.-\langle n\rangle^{2}\right) /\langle n\rangle$ is a measure of the intensity fluctuations. For Poissonian photon statistics, $F=1$. For thermal photon statistics, $F=1$ $+\langle n\rangle$. All plotted quantities are dimensionless.

photon numbers appearing in Eq. (16) [remember that $k \neq 0$ in Eq. (16)]. Then the device becomes an ideal thresholdless laser with Poissonian photon statistics for all pump powers, given by $p_{n}=\exp \left(-N_{\mathrm{ex}} / 2\right)\left(N_{\mathrm{ex}} / 2\right)^{n} / n$ !

Figure 1 shows the result of a numerical calculation using the exact Eq. (16) both in (a) the ordinary laser regime and in (b) the ideal thresholdless regime. We were careful to choose experimentally realistic values of the parameters and not to violate the assumptions that there is only a single atom at a time in the cavity and that cavity damping can be neglected during the interaction between each atom and the field. The first assumption requires $\tau<t_{\mathrm{at}}$, where $t_{\mathrm{at}}=1 / R$ is the average time that elapses from the arrival of an atom in the cavity to the arrival of the next atom. The second assumption requires $\tau \ll 1 / \kappa$. For a typical vacuum Rabi frequency of $\pi$ $\times 50 \mathrm{kHz}$, the value of $W$ adopted in (a) implies an interaction time of about $15 \mu \mathrm{s}$ (corresponding to an atomic velocity of about $700 \mathrm{~m} / \mathrm{s}$ ) which is much shorter than the photon lifetime (about $1 \mathrm{~ms}$ ), satisfying the second assumption. As $N_{\mathrm{ex}}=1 /\left(\kappa t_{\mathrm{at}}\right)$, the first assumption is satisfied only for $N_{\mathrm{ex}}$ $\ll 66.66$. This together with the experimental limitations on the atomic beam explains why the maximum value of $N_{\mathrm{ex}}$ in this plot is 50. In (b), the interaction time is longer, about 70 $\mu \mathrm{s}$ (corresponding to a velocity of about $150 \mathrm{~m} / \mathrm{s}$ ) but still much shorter than the photon lifetime, satisfying the second assumption. The maximum attainable value of $N_{\mathrm{ex}}$ is just about 14, otherwise there will be more than one atom at a time in the cavity, violating the first assumption; nevertheless, we have varied $N_{\text {ex }}$ up to 50 in (b) to facilitate the comparison with (a).

To understand the physics behind these results, we must first analyze $\beta$. In ordinary lasers where the gain medium is kept inside the cavity all the time, $\beta$ is given by $[12,13]$

$$
\beta=\frac{2 g^{2} /\left(\gamma_{\perp}+2 \kappa\right)}{2 g^{2} /\left(\gamma_{\perp}+2 \kappa\right)+\gamma_{\|} / 2}
$$

From Eq. (19), we see that (as $\gamma_{\|}<\gamma_{\perp}+2 \kappa$ ) a necessary condition for $\beta \rightarrow 1$ is $g \gg \gamma_{\|}$[14]. Notice that $g$ does not have to be larger than $\gamma_{\perp}$ and $\kappa$. In fact, it can be easily checked that for $\beta$ to approach 1 it is sufficient that $g / \gamma_{\|}$be such a large number that $\left(g / \gamma_{\|}\right) g \gg \gamma_{\perp}, \kappa$. Here, as in an ordinary laser, spontaneous emission is irreversible with its rate in the cavity mode given by $4 g^{2} / \gamma_{\perp}$ in the limit of $\gamma_{\perp}$ $\gg g$. On the other hand, unlike an ordinary laser, we assume that $\left(g / \gamma_{\|}\right) g \gg \gamma_{\perp}$, which together with $g \gg \gamma_{\|}$and $g \gg \kappa$ (the ideal thresholdless requirement) guarantees that virtually all spontaneous emission photons will go into the cavity mode when the atom is inside the cavity. In that case, according to Eq. (19) $\beta$ should reach its maximum value, which for the present case, with polarization decay only, is $1 / 2$ (see Sec. II). But as every atom will eventually leave the cavity, $\beta$ is not simply given by Eq. (19). There is an extra loss channel: some spontaneous emission photons will be emitted into external free-space modes by atoms that failed to emit while crossing the cavity. This is why the laser can go from ordinary threshold operation to the ideal thresholdless regime when the speed at which the atoms cross the cavity is varied: at speeds slow enough for the crossing time $t_{\text {int }}$ to be much longer than the inverse of the spontaneous emission rate, $\beta$ will be very close to its maximum value of $1 / 2$, while at high speeds $\beta$ will be much smaller than $1 / 2$.

We should stress that this is an ideal thresholdless laser where $\beta=1 / 2$ rather than 1 , as can be seen by noticing that the slope of the input-output curve is one-half both in the case of the thresholdless regime as well as in ordinary operation above threshold. The fact that thresholdless behavior is at all possible with $\beta=1 / 2$ instead of $\beta=1$ is a nice example of a point that was made in Ref. [4]: In the strong coupling regime, outside the thermodynamic limit, there is no longer the sort of universality that holds in the thermodynamic limit where true thresholds can exist.

\section{STOCHASTICITY IN THE INTERACTION TIME}

Another way to introduce stochasticity is in fact well known [15,3]: A spread in the interaction time will turn the micromaser into a laser [3]. What was not realized, apparently, is that at very low cavity temperatures, where the average number of thermal photons is negligible, the micromaser would become an ideal thresholdless laser. Assuming a Gaussian distribution of the interaction time with mean $\bar{\tau}$ and rms spread $\sigma$ as in Ref. [3], when the average number of thermal photons is negligible, the probability of finding $n$ photons in the cavity mode at the "steady state" of the micromaser (with Poissonian pumping) for $n>0$ is given by [3]

$$
p_{n}=p_{0} \prod_{k=1}^{n} \frac{N_{\mathrm{ex}}}{2 k}\left\{1-e^{-k(g \sigma)^{2} / 2} \cos (2 \sqrt{k} g \bar{\tau})\right\}
$$

where $N_{\text {ex }}$ is the average number of atoms that cross the cavity within a photon lifetime and $p_{0}$ is the probability of finding no photons in the cavity mode, which can be determined as a normalization constant from the condition $\Sigma p_{n}$ $=1$. Now if $(g \sigma)^{2} \gg 1$, this probability $p_{n}$ becomes

$$
p_{n}=p_{0}\left(\frac{N_{\mathrm{ex}}}{2}\right)^{n} \frac{1}{n !} .
$$

Noticing that $N_{\text {ex }}$ is the counterpart, in the micromaser, of the number of excited atoms of the gain medium in an ordinary laser, which is a measure of the pumping power, we see that 

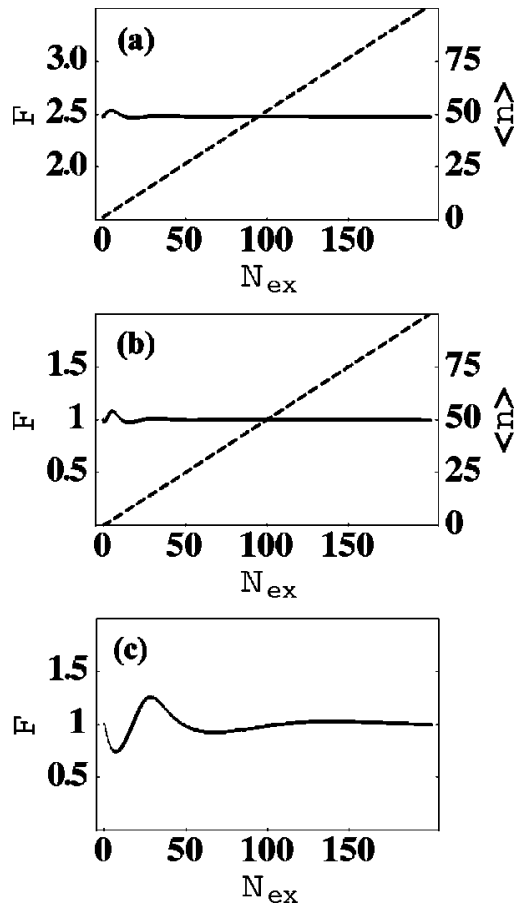

FIG. 2. The Fano parameter (full line) and the average photon number (dotted line) as functions of $N_{\mathrm{ex}}$ for a realistic thermal velocity distribution of a beam of ${ }^{85} \mathrm{Rb}$ Rydberg atoms from a $433 \mathrm{~K}$ oven which go through a high- $Q$ cavity kept at $2 \mathrm{~K}$ (a) and at 0.15 $\mathrm{K}$ (b). Both figures refer to the strong maser transition $63 P_{3 / 2} \leftrightarrow 61 D_{5 / 2}$ of ${ }^{85} \mathrm{Rb}$ for which the vacuum Rabi frequency is 44 $\mathrm{kHz}$. In (c) the cavity temperature is the same as in (b), but the vacuum Rabi frequency that is taken as $20 \mathrm{kHz}$ corresponding to the weak maser transition $63 P_{3 / 2} \leftrightarrow 61 D_{3 / 2}$ of ${ }^{85} \mathrm{Rb}$. All plotted quantities are dimensionless.

the photon statistics will be Poissonian for any pumping, thus realizing the ideal thresholdless regime.

This general conclusion still holds when the artificial assumption of a Gaussian distribution of the interaction time is dropped in favor of a more realistic thermal velocity distribution [16] given by

$$
f(v)=2\left(\frac{m}{2 k T}\right)^{2} v^{3} e^{-v^{2} m /(2 k T)} N_{0},
$$

where $v$ is the atomic velocity, $m$ is the mass of the atom, $T$ is the temperature of the oven, and $N_{0}$ is just a normalization constant. The counterpart of Eq. (20), with the Gaussian replaced by Eq. (22), is given by

$$
p_{n}=p_{0} \prod_{k=1}^{n} \frac{N_{\mathrm{ex}}}{k} \int d v f(v) \sin ^{2}\left(g \frac{L}{v} \sqrt{k}\right)
$$

where $L$ is the cavity length.

In Fig. 2, we plot the result of a numerical calculation of the average photon number and the Fano parameter as func- tions of the pumping $N_{\mathrm{ex}}$ with Eq. (20) replaced by Eq. (23). We have used values of $m$ and $T$ appropriate for a beam of ${ }^{85} \mathrm{Rb}$ atoms coming straight out of an oven at $433 \mathrm{~K}$, without any velocity selection. For the cavity length $L$ we have adopted the typical experimental value of $24 \mathrm{~mm}$ corresponding to the closed microwave cylindrical cavities used in micromaser experiments. As the photon lifetime currently achievable in such closed cavities is as long as $0.2 \mathrm{~s}$, the criterion $\tau \ll 1 / \kappa$ is easily satisfied. Similarly, the criterion for having a single atom at a time in the cavity, $\tau N_{\mathrm{ex}}<1 / \kappa$, is also satisfied even for values of $N_{\text {ex }}$ well above 200 .

Figure 2(a) shows that at the lowest cavity temperature attained in the first micromaser experiment [2], $2 \mathrm{~K}$, the thermal photons do not allow the Fano parameter to approach 1. However, we see in Fig. 2(b) that at the presently [17] lowest temperature of $0.15 \mathrm{~K}$, apart from a little bump near the origin, the Fano parameter is always 1 (i.e., Poissonian photon statistics) showing no trace of threshold, as predicted by the simplified analytic theory, i.e., Eqs. (20) and (21). The little bump near the origin is not a signature of threshold, but a residue of the imperfect averaging of the interaction time. Even without any velocity selection, the distribution of interaction times still has a well-defined peak whose width is narrow enough to maintain some of the coherent effects of the lowest Rabi oscillations (i.e., for low photon numbers, near the origin of the curve). This is clear from Fig. 2(c), which shows that the bump increases for smaller vacuum Rabi frequencies as these should be better resolved by the peak in the distribution of interaction times.

\section{CONCLUSIONS}

We have shown that a laser, where only the atomic polarization decay is non-negligible in comparison with the vacuum Rabi frequency, can be made to operate in a regime with an ordinary laser threshold or one without any threshold. In the latter regime, the photon statistics remains Poissonian for arbitrarily small pump powers, characterizing what has been called ideal thresholdlessness. However, this is a very peculiar ideal thresholdless laser where $\beta=1 / 2$ rather than 1. It is an example of the loss of universality and the disappearance of true phase transitions outside the thermodynamic limit, in the strong coupling regime of a cavity QED laser [4]. Even though the large vacuum Rabi frequency required for this is not yet achievable in the optical regime, the current state of the art in cavity QED microwave experiments readily provides $g \gg \gamma_{\|}, \kappa$ and temperatures as low as $0.15 \mathrm{~K}$ [17] where the average number of thermal photons is negligible. This allows an experimental proof-ofprinciples realization of such a device in the microwave domain.

\section{ACKNOWLEDGMENT}

This work was supported by the "Stichting voor Fundamenteel Onderzoek der Materie (FOM)." 
[1] E. T. Jaynes and F. W. Cummings, Proc. IEEE 51, 89 (1963); B. W. Shore and P. L. Knight, J. Mod. Opt. 40, 1195 (1993).

[2] D. Meschede, H. Walther, and G. Müller, Phys. Rev. Lett. 54, 551 (1985).

[3] P. Filipowicz, J. Javanainen, and P. Meystre, Phys. Rev. A 34, 3077 (1986).

[4] P. R. Rice and H. J. Carmichael, Phys. Rev. A 50, 4318 (1994).

[5] M. P. van Exter, G. Nienhuis, and J. P. Woerdman, Phys. Rev. A 54, 3553 (1996).

[6] H. Yokoyama and S. D. Brorson, J. Appl. Phys. 66, 4801 (1989); H. Yokoyama, Science 256, 66 (1992).

[7] G. Björk and Y. Yamamoto, IEEE J. Quantum Electron. 27, 2386 (1991); G. Björk, A. Karlsson, and Y. Yamamoto, Phys. Rev. A 50, 1675 (1994).

[8] See, for example, H. J. Kimble, in Cavity Quantum Electrodynamics, edited by P. R. Berman (Academic Press, Boston, 1994), pp. 203-266; S. Haroche, in Fundamental Systems in Quantum Optics, Proceedings of the Les Houches Summer School of The Theoretical Physics, Les Houches, 1990, edited by J. Dalibard, J.-M. Raimond, and J. Zinn-Justin (NorthHolland, Amsterdam, 1992), pp. 767-940.

[9] M. Brune, F. Schmidt-Kaler, A. Maali, J. Dreyer, E. Hagley, J. M. Raimond, and S. Haroche, Phys. Rev. Lett. 76, 1800 (1996).

[10] See, for example, P. Meystre and M. Sargent, Elements of
Quantum Optics (Springer-Verlag, Berlin, 1991).

[11] H.-J. Briegel and B.-G. Englert, Phys. Rev. A 47, 3311 (1993).

[12] I. Protsenko, P. Domokos, V. Lefèvre-Seguin, J. Hare, J. M. Raimond, and L. Davidovich, Phys. Rev. A 59, 1667 (1999).

[13] B. Jones, S. Ghose, J. P. Clemens, P. R. Rice, and L. M. Pedrotti, Phys. Rev. A 60, 3267 (1999).

[14] This is only a necessary condition, not a sufficient one. In fact, a laser satisfying $g \gg \gamma_{\|}$but with $\beta=2 \times 10^{-5}$ has been studied recently by Y. Lien, S. M. de Vries, N. J. van Druten, M. P. van Exter, and J. P. Woerdman, Phys. Rev. Lett. 86, 2786 (2001). This laser operates in a curious regime where there is a very well-defined kink in the input-output curve, but the intensity noise shows almost no trace of a laser threshold remaining at the level of thermal light up to very high pumping powers. For a nice review on this and other surprises regarding quantum noise of small lasers, see J. P. Woerdman, M. P. van Exter, and N. J. van Druten, Adv. At., Mol., Opt. Phys. 47, 205 (2001).

[15] W. E. Lamb, Jr., in Lectures in Theoretical Physics, edited by W. E. Brittin and B. W. Downs (Interscience, New York, 1960), Vol. II.

[16] N. F. Ramsey, Molecular Beams (Clarendon Press, Oxford, 1956).

[17] M. Weidinger, B. T. H. Varcoe, R. Heerlein, and H. Walther, Phys. Rev. Lett. 82, 3795 (1999). 